\title{
Article \\ An Automatic Immunoaffinity Pretreatment of Deoxynivalenol Coupled with UPLC-UV Analysis
}

\author{
Hongmei Liu ${ }^{1}$, Zhihong Xuan ${ }^{1}$, Jin Ye ${ }^{1, *}{ }^{\oplus}$, Jinnan Chen ${ }^{1}$, Meng Wang ${ }^{2}\left(\mathbb{D}\right.$, Stephan Freitag ${ }^{3}$, Rudolf Krska ${ }^{3,4}{ }^{\oplus}$, \\ Zehuan Liu ${ }^{1},{ }^{1}, \mathrm{Li} \mathrm{Li}^{1}, \mathrm{Yu} \mathrm{Wu}{ }^{1}$ and Songxue Wang ${ }^{1, *}$
}

1 Academy of National Food and Strategic Reserves Administration, No. 11 Baiwanzhuang Street, Xicheng District, Beijing 100037, China; Ihm@ags.ac.cn (H.L.); xzh@ags.ac.cn (Z.X.); cjn@ags.ac.cn (J.C.); haiyangzhixin2006@163.com (Z.L.); 11@ags.ac.cn (L.L.); wyu@ags.ac.cn (Y.W.)

2 Institute of Quality Standard and Testing Technology, Beijing Academy of Agriculture and Forestry Sciences, Beijing 100097, China; wangm@brcast.org.cn

3 Institute of Bioanalytics and Agro-Metabolomics, Department of Agrobiotechnology (IFA-Tulln), University of Natural Resources and Life Sciences Vienna (BOKU), 3430 Tulln, Austria; stephan.freitag@boku.ac.at (S.F.); rudolf.krska@boku.ac.at (R.K.)

4 Institute for Global Food Security, School of Biological Sciences, Queen's University Belfast, Belfast BT9 5DL, Northern Ireland, UK

5 College of Food Science and Engineering, Central South University of Forestry and Technology, Changsha 410004, China

* Correspondence: yj@ags.ac.cn (J.Y.); wsx@ags.ac.cn (S.W.); Tel.: +86-010-5645-2662 (J.Y.); +86-010-5645-2668 (S.W.)

check for updates

Citation: Liu, H.; Xuan, Z.; Ye, J.; Chen, J.; Wang, M.; Freitag, S.; Krska, R.; Liu, Z.; Li, L.; Wu, Y.; et al. An Automatic Immunoaffinity Pretreatment of Deoxynivalenol Coupled with UPLC-UV Analysis. Toxins 2022, 14, 93. https://doi.org/ $10.3390 /$ toxins 14020093

Received: 30 November 2021

Accepted: 6 January 2022

Published: 25 January 2022

Publisher's Note: MDPI stays neutral with regard to jurisdictional claims in published maps and institutional affiliations.

Copyright: (C) 2022 by the authors. Licensee MDPI, Basel, Switzerland. This article is an open access article distributed under the terms and conditions of the Creative Commons Attribution (CC BY) license (https:// creativecommons.org/licenses/by/ $4.0 /)$.

\begin{abstract}
An immunoaffinity magnetic beads (IMBs) based automatic pretreatment method was developed for the quantitative analysis of deoxynivalenol (DON) by ultra-performance liquid chromatography and ultraviolet detector (UPLC-UV). First, N-hydroxysuccinimide-terminated magnetic beads (NHS-MBs) with good magnetic responsivity and dispersibility were synthesized and characterized by optical microscopy, scanning electron microscopy (SEM), and laser diffraction-based particle size analyzer. Then, the amino groups of anti-DON monoclonal antibody $(\mathrm{mAb})$ and the NHS groups of NHS-MBs were linked by covalent bonds to prepare IMB, without any activation reagent. The essential factors affecting the binding and elution of DON were meticulously tuned. Under optimal conditions, DON could be extracted from a real sample and eluted from IMB by water, enabling environmentally friendly and green analysis. Hence, there was no need for dilution or evaporation prior to UPLC-UV analysis. DON in 20 samples could be purified and concentrated within $30 \mathrm{~min}$ by the mycotoxin automated purification instrument (MAPI), allowing for automated, green, high-throughput and simple clean-up. Recoveries at four distinct spiking levels in corn and wheat ranged from $92.0 \%$ to $109.5 \%$ with good relative standard deviations (RSD, $2.1-7.0 \%$ ). Comparing the test results of IAC and IMB in commercial samples demonstrated the reliability and superiority of IMB for quantitatively analyzing massive samples.
\end{abstract}

Keywords: deoxynivalenol; immunoaffinity magnetic beads; automatic; UPLC-UV

Key Contribution: An immunoaffinity magnetic beads-based automatic pretreatment method was developed for the quantitative analysis of deoxynivalenol by UPLC-UV, with water used as the solvent for extraction and elution.

\section{Introduction}

The primary representative of the type B trichothecenes, deoxynivalenol (DON), commonly called vomitoxin, is generated by some Fusarium species [1]. DON is one of the most prevalent mycotoxins in various cereals crops and processed grains, such as corn, wheat, bread, beer, and malt [2-5], with a detection rate of 57\% in 11,444 tested samples [6]. The consumption of grains or food products contaminated with DON could result in various 
adverse effects to humans and animals, including feed refusal, gastroenteritis, nutrient malabsorption, diarrhea, vomiting, teratogenicity, immunotoxicity and cardiotoxicity [7-9]. The International Agency for Research on Cancer has categorized DON in the group 3 as not classifiable regarding their carcinogenicity to humans since 1993 [8]. Thus, many countries have established the maximum level (ML) for DON in cereals and its derived products. The ML of DON in Commission Regulation (EC) No 1881/2006 ranged from $200 \mu \mathrm{g} / \mathrm{kg}$ (processed cereal-based foods and baby foods for infants and young children) to $1750 \mu \mathrm{g} / \mathrm{kg}$ (unprocessed durum wheat, oats and maize) [10]. The ML of DON in China was $1000 \mu \mathrm{g} / \mathrm{kg}$ in cereals and related products [11]. In addition, the Joint FAO/WHO Expert Committee on Food Additives recommended a daily dose of $1 \mathrm{mg} / \mathrm{kg}$ body weight. Furthermore, a tolerable maximum daily dose of $1 \mathrm{mg}$ DON per kilogram of body weight was recommended by the Joint FAO/WHO Expert Committee on Food Additives [12].

DON, a polar organic compound, is stable in processing and cooking, thus to avoid the potential health risks it is important to evaluate its contamination levels [13]. Highperformance liquid chromatography (HPLC) with ultraviolet (UV) $[3,13,14]$ or mass spectrometry (MS) $[7,15]$ and gas chromatography with electron capture [16] or MS detection [17] were the most prevalent approaches for the quantitative assessment of DON in food and feed. To eliminate interfering matrix chemicals, concentrate DON and protect the analytical instrument, various clean-up approaches have been utilized after the solid-liquid extraction of DON, such as dispersive solid-phase extraction (DSPE) [15] immunoaffinity columns (IACs) $[2,3,5,13,14]$ or multifunctional clean-up columns [18]. Among them, the IAC, on the basis of the selective interaction between DON and its antibodies, is one of the most popular and effective sample pretreatment methods. However, using IAC as a pretreatment method is less well-adapted to high-throughput screening of DON because it necessitates tedious and time-consuming operation procedures, expensive use costs, and highly skilled technicians.

With the increasing desire to improve pretreatment efficiency and reduce testing costs, major efforts have been dedicated to high-throughput and automatic sample pretreatment methods. Immunoaffinity magnetic beads (IMBs), with magnetic beads (MBs) as carriers of specific antibodies, provide an alternative immunoaffinity separation technique to conventional IACs, due to the high surface-to-volume ratio and superparamagnetism of the IMBs [19]. Compared with IACs, IMBs have excellent dispersibility in a liquid medium, which increases the opportunities for the interaction with their targets. Hence, IMBs can be used to simply, rapidly and specifically capture and separate targets from complex sample matrices through an exterior magnetic field, with no need for centrifugation or filtration [20]. The applicability of IMB has been widely demonstrated in diagnostic techniques [21] and protein purification [22]. Furthermore, an automated and high-throughput sample pretreatment method for ochratoxin A and aflatoxins was built on the basis of their corresponding IMBs in our previous works $[23,24]$. Although the detection level and rate of DON were much higher than those of ochratoxin A and aflatoxins $[4,6]$, the pretreatment of DON has yet to be automated for high throughput.

In the present study, an automated and high-throughput pretreatment method was developed for highly efficient and selective isolation and concentration of DON on the basis of anti-DON IMB. N-hydroxysuccinimide-terminated magnetic beads (NHS-MBs) with good magnetic responsivity and dispersibility were synthesized and used as carriers of the anti-DON monoclonal antibody $(\mathrm{mAb})$, rather than the conventional agarose in IACs and the traditional $\mathrm{MB}$ with carboxyl or amino groups. The factors affecting the incubation with the IMBs and elution of DON from the IMBs were meticulously tuned for efficient and selective isolation and concentration of DON. The mycotoxin automatic purification instrument (MAPI) was used to achieve high-throughput, easy and automatic sample pretreatment, with its 20 pairs of magnetic sticks and plastic coats. Water was used to extract DON from naturally contaminated samples [2,3], as well as to elute DON from IMBs instead of methanol and acetonitrile, enabling environmental safe and green analysis. Hence, there is no need for dilution or evaporation of the eluent prior to UPLC-UV analysis, thereby 
further improving sample handling efficiency. In the end, the applicability of IMBs for simple, green, automated and high-throughput clean-up and the subsequent determination of DON by UPLC-UV was demonstrated by analyzing naturally contaminated samples and DON reference materials.

\section{Results and Discussion}

\subsection{Characterization of the $M B$ s and IMBS}

SEM was first used to characterize the geometry and morphology of $\mathrm{Fe}_{3} \mathrm{O}_{4}, \mathrm{Fe}_{3} \mathrm{O}_{4} \cdot \mathrm{SiO}_{2}$, and IMB. Compared with Figure $1 \mathrm{a}\left(\mathrm{Fe}_{3} \mathrm{O}_{4}\right)$, the surface of $\mathrm{Fe}_{3} \mathrm{O}_{4}$ was coated with a smooth layer of silica, as shown in Figure 1b, which could improve hydrophilicity, dispersion and stability [20]. The SEM image in Figure 1c of the IMB exhibited excellent monodispersity. The core of the IMB was the clusters of $\mathrm{Fe}_{3} \mathrm{O}_{4} \cdot \mathrm{SiO}_{2}$ in Figure 1d, which were uniformly entrapped within the agarose hydrogel either in an aggregated or dispersed form to further enhance the hydrophilicity and stability of the IMB. The size of the IMB in Figure 1e was in the range of $5 \mu \mathrm{m}$ to $25 \mu \mathrm{m}$, most of which was approximately $15 \mu \mathrm{m}$, detected a by laser diffraction-based particle size analyzer. Because of the clusters of $\mathrm{Fe}_{3} \mathrm{O}_{4} \cdot \mathrm{SiO}_{2}$, the scattered IMBs in an external magnetic field could accumulate within $20 \mathrm{~s}$ (Figure 1f), before dispersing quickly with minor shaking once the magnetic field was removed, confirming its superior magnetic responsivity and dispersibility.

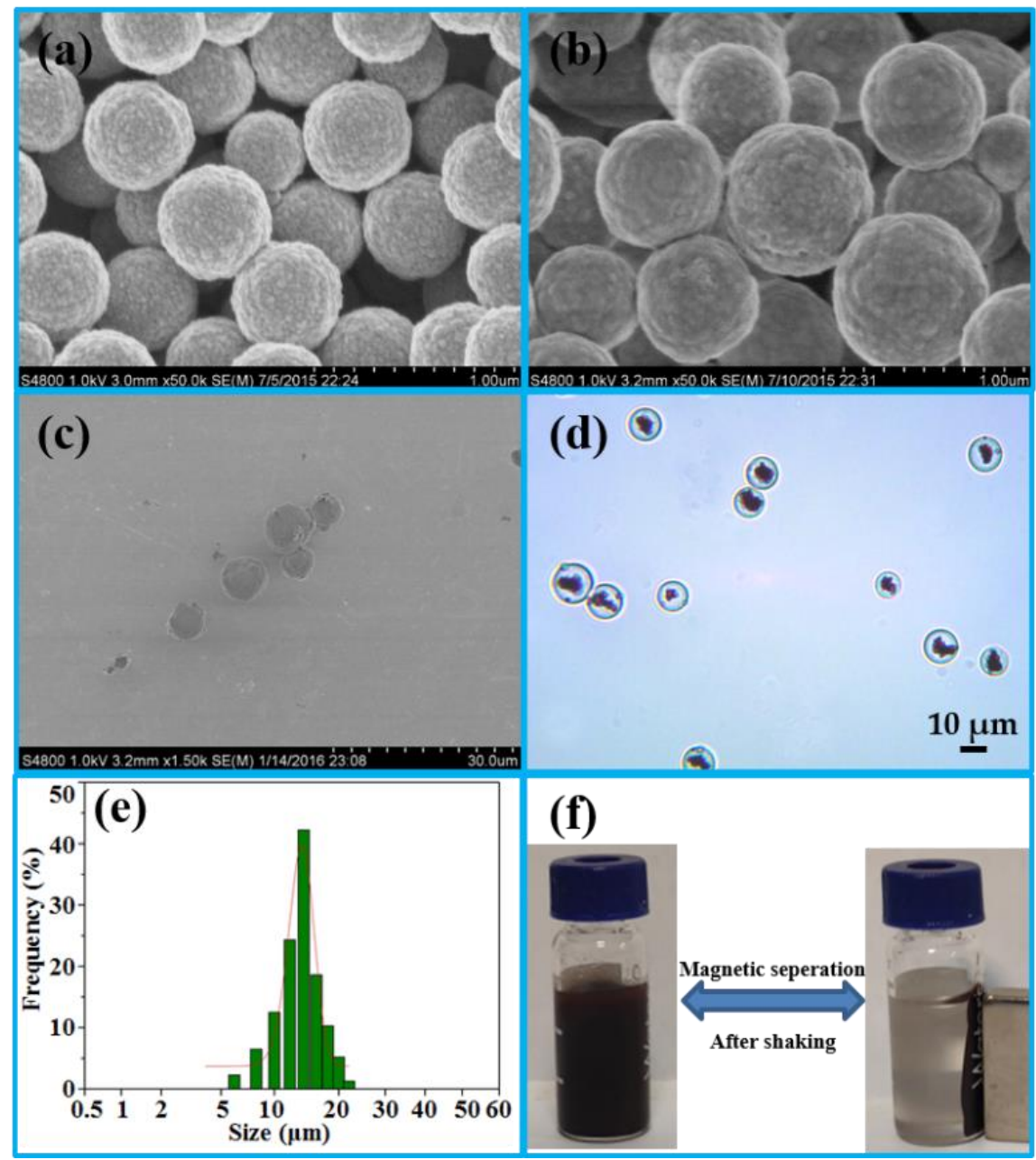

Figure 1. SEM images of $\mathrm{Fe}_{3} \mathrm{O}_{4}(\mathbf{a}), \mathrm{Fe}_{3} \mathrm{O}_{4} \cdot \mathrm{SiO}_{2}$ (b) and IMB (c); optical microscopy picture of the IMB (d); size distribution graph of the IMB (e); paramagnetism of the IMB (f). (SEM: scanning electron microscopy; IMB: immunoaffinity magnetic beads). 
The content of NHS on the surface of NHS-MB was quantified based on a spectrophotometric assay, according to a previous report [25]. The principle for the assay was that N-hydroxysuccinimide was ionized under $0.1 \mathrm{M} \mathrm{NH}_{4} \mathrm{OH}$ basic conditions, whose absorption spectrum at $260 \mathrm{~nm}$ was increased. Firstly, a series of NHS standard solutions $(0.2 \mathrm{mM}$, $0.5 \mathrm{mM}, 1.0 \mathrm{mM}, 1.2 \mathrm{mM}, 1.4 \mathrm{mM}, 1.8 \mathrm{mM}, 2.0 \mathrm{mM}$ ) was configured by $0.1 \mathrm{M} \mathrm{NH}_{4} \mathrm{OH}$, whose absorbance was measured in triplicate at $260 \mathrm{~nm}$. The concentration $(x)$ of NHS and absorbance at $260 \mathrm{~nm}(y)$ were used to draw the standard curve $(y=0.6583 x+0.051$, with $\mathrm{R}^{2}=0.9981$ ). Secondly, to prevent the rapid hydrolysis of NHS, $1 \mathrm{~mL}$ of NHS-MB $(10 \%, v / v)$ was quickly washed twice with water with the aid of a magnetic stand. Then, the NHS-MB was mixed vertically and incubated with $1 \mathrm{~mL}$ of $\mathrm{NH}_{4} \mathrm{OH}(0.1 \mathrm{M})$ for $5 \mathrm{~min}$ at room temperature. After magnetic separation, the supernatant was transferred into another centrifuge tube. The supernatant was then withdrawn into the aforementioned centrifuge tube, after another $1 \mathrm{~mL}$ of $\mathrm{NH}_{4} \mathrm{OH}(0.1 \mathrm{M})$ was added and mixed vertically with $\mathrm{MB}$ for $5 \mathrm{~min}$. After diluting the supernatant to $5 \mathrm{~mL}$ with $\mathrm{NH}_{4} \mathrm{OH}(0.1 \mathrm{M})$, the average absorbance was 1.101 at $260 \mathrm{~nm}$ in triplicate. Hence, $8 \mu \mathrm{mol}$ of NHS was on the surface of $1 \mathrm{~mL}$ of NHS-terminated MB $(10 \%, v / v)$, calculated by the standard curve of NHS $(\mathrm{NHS}(\mu \mathrm{mol})=(1.101-0.048) / 0.6583 \times 5=8 \mu \mathrm{mol})$.

\subsection{Synthesis of the $I M B$}

\subsubsection{Screening of the $\mathrm{mAb}$}

The high affinity of the $\mathrm{mAb}$ was another core for the IMB, which was the identification component of DON. As a carrier for mAb, NHS-MB can couple with anti-DON mAb at room temperature through the covalent bond between NHS groups on the MB and amino groups on the $\mathrm{mAb}$, without the activation of EDC or glutaraldehyde. Firstly, antibodies from different manufacturers were screened by comparing their recovery rate and the maximum adsorption capacity. Different IMBs were prepared on the basis of $400 \mu \mathrm{g} \mathrm{mAb}$ from four manufacturers (A: Wuhan Yangda Biological Technology Co., Ltd., Wuhan, China; B: Shandong Landu Biotechnology Co., Ltd., Shandong, China; C: Wuxi Chuangpu Biological Technology Co., Ltd., Wuxi, China and D: Beijing Huaanmaike Biotechnology Co., Ltd., Beijing, China) and $2 \mathrm{mg}$ NHS-terminated MB, according to the procedure in "4.5 Preparation of Anti-DON Immunoaffinity Magnetic Beads". Then, 100 ng of DON was added to the first well to investigate the recovery rate of the IMB, while $500 \mathrm{ng}$ of DON was used to assess its maximum adsorption capacity. The recovery rates for the IMB from the $\mathrm{mAbs}$ of $\mathrm{C}$ and $\mathrm{D}$ were close to $100 \%$, whereas those for $\mathrm{A}$ and $\mathrm{B}$ were lower than $40 \%$, as shown in Figure 2a. The maximum adsorption capacity in Figure $2 b$ was $C>D>A>B$. Hence, the $\mathrm{mAb}$ from $\mathrm{C}$ was selected in the subsequent experiments.

\subsubsection{Optimization of the Coupling Conditions}

The IMB was transferred between the different wells of the kit with the aid of magnetic bars. Hence, too many MBs may affect its transfer efficiency due to the limitations of well size and magnetic bars, and an insufficient amount of MBs could lead to a low $\mathrm{mAb}$ load. Because of the high ML (1000 $\mu \mathrm{g} / \mathrm{kg}$ ) of the DON, the low mAb load could result in the maximum adsorption capacity of IMB not meeting the requirements of actual sample detection. Therefore, to circumvent the limitations of $\mathrm{MB}$ concentration and $\mathrm{mAB}$ load, a defined amount of MBs (5 mg) was used for coupling different amounts of antibodies $(200,300,400,500,600,800$ and $1000 \mu \mathrm{g})$. Then, $1500 \mathrm{ng}$ of DON was applied to investigate the maximum adsorption capacity of $2 \mathrm{mg}$ IMB. The DON in the incubation well and in the eluting well of the kit was analyzed by UPLC-UV after treatment with the MAPI. The detected amount of the DON was shown in Figure 2c. With the increase in the amount of $\mathrm{mAb}$, less DON was left in the incubation well (the first well), while more DON was eluted in the elution well (the seventh well). Increasing the amount of antibody could further increase the maximum adsorption capacity of IMB, which could lead to the wasting of $\mathrm{mAb}$. The maximum adsorption capacity was $670 \mathrm{ng}$, which was when $1000 \mu \mathrm{g}$ of $\mathrm{mAb}$ and $0.5 \mathrm{~mL}$ MB were used to prepare the IMB. When $1 \mathrm{~mL}$ of the supernatant in "4.6. Sample 
sources and preparation" was added in first well of the kit, the maximum amount of DON in the real sample that could be accurately quantified was $2680 \mu \mathrm{g} / \mathrm{kg}$, which was much higher than its MLs $(1000 \mu \mathrm{g} / \mathrm{kg})$.
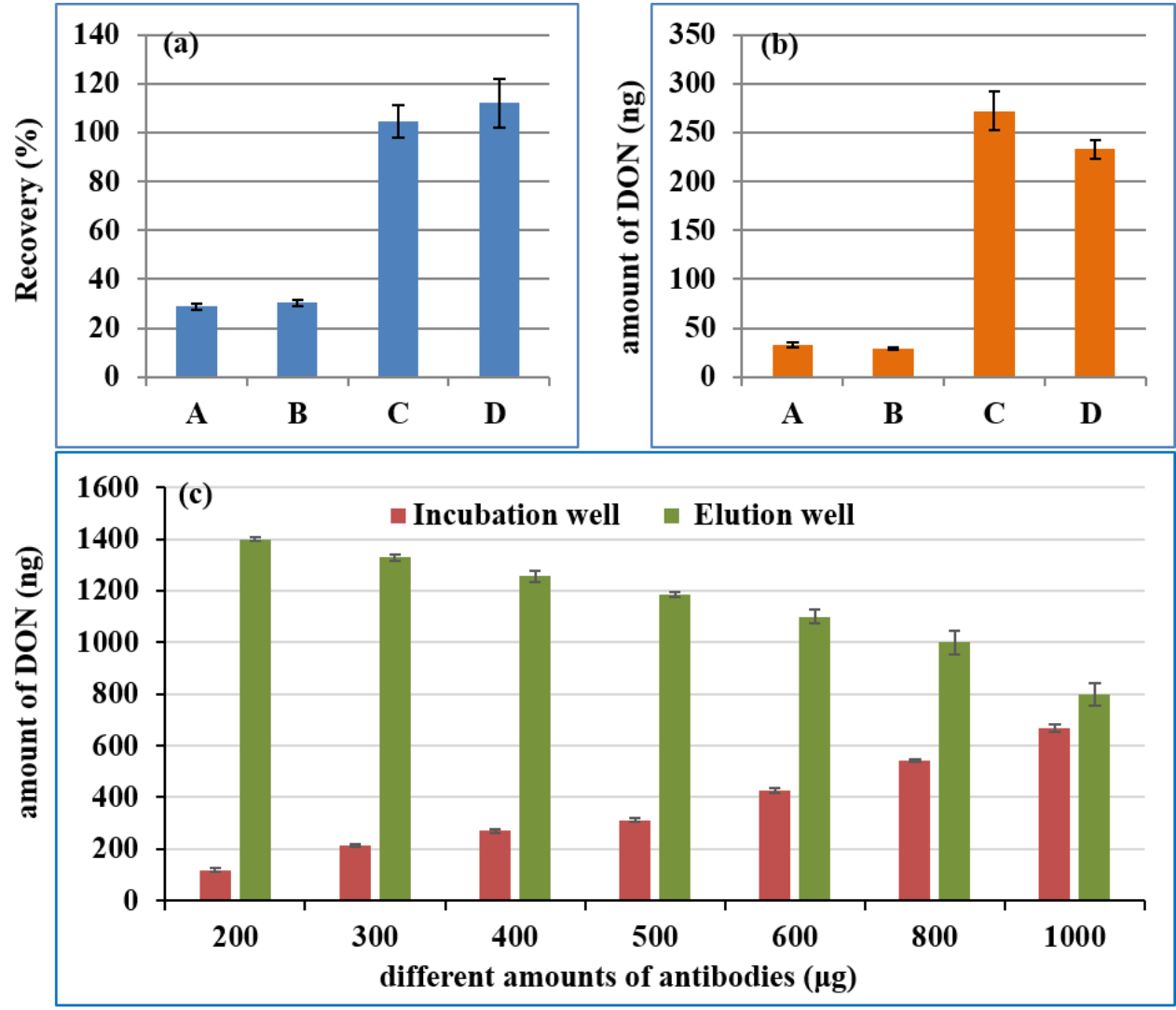

Figure 2. The recovery of $100 \mathrm{ng} \mathrm{DON}$ (a), the maximum adsorption capacity (b) of IMBs from different manufacturers, and the maximum adsorption capacity (c) of the IMB by coupling different amounts of antibodies with $0.5 \mathrm{~mL}$ MB. (A: Wuhan Yangda Biological Technology Co., Ltd.; B: Shandong Landu Biotechnology Co., Ltd.; C: Wuxi Chuangpu Biological Technology Co., Ltd. and D: Beijing Huaanmaike Biotechnology Co., Ltd.; DON: deoxynivalenol; IMB: immunoaffinity magnetic bead; MB: magnetic bead).

\subsection{Optimization of the Elution Conditions}

Adequate time was necessary for the IMB to fully capture the DON. Hence, $100 \mathrm{ng}$ of DON was added to the first well and then incubated for $0.5,1,2,5,8$, and 10 min with $2 \mathrm{mg}$ IMBs. The recovery in the elution well is shown in Figure $3 \mathrm{a}$. The recovery of DON improved as incubation time increased within $5 \mathrm{~min}$, and fluctuated around 100\% from $5 \mathrm{~min}$ to $10 \mathrm{~min}$. Hence, it only takes $5 \mathrm{~min}$ for the IMB to completely capture the DON, which was used as the incubation time in the subsequent experiments. 

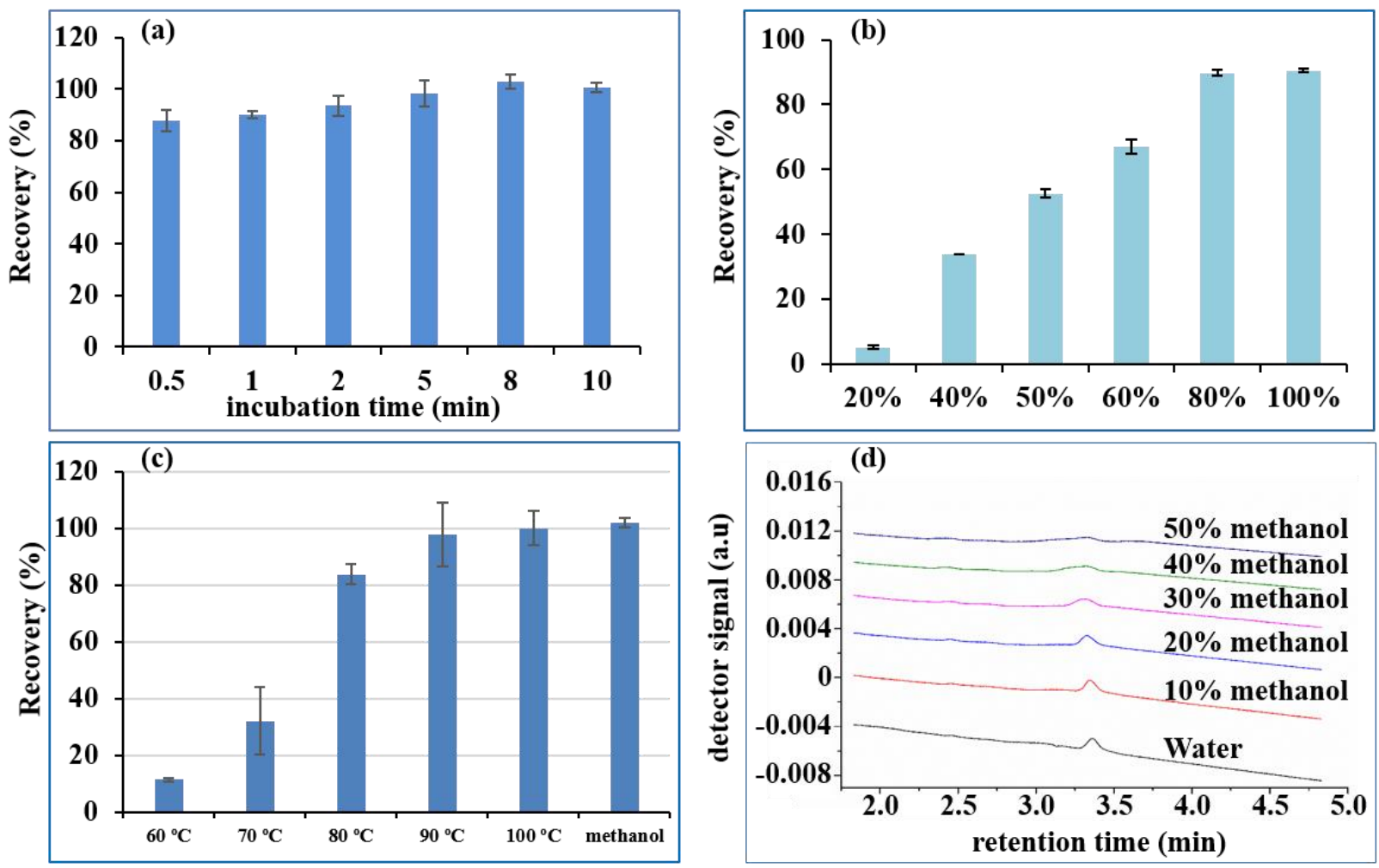

Figure 3. Recovery of $100 \mathrm{ng}$ DON under different incubation times (a), methanol concentration in eluent (b), water at different temperatures in elution (c) and chromatogram of $100 \mathrm{ng} / \mathrm{mL} \mathrm{DON} \mathrm{in}$ different concentrations of methanol (d). (DON: deoxynivalenol).

\subsection{Optimization of the Elution Conditions}

\subsubsection{Elution Ability of Different Concentrations of Methanol}

The most commonly employed eluent for the IAC of mycotoxins is methanol $[2,5,13,14]$, which can deactivate mAbs and desorb the target analytes from IAC. Therefore, we firstly investigated the elution ability of different concentrations of methanol in water. Different ratios of methanol-water $(20+80$ to $80+20)$ were therefore used as the eluent solvent for $2 \mathrm{mg}$ IMBs loaded with $100 \mathrm{ng}$ of DON, to optimize the lowest concentration of methanol in the eluent solvent. As seen in Figure 3b, increasing the methanol concentration from $20 \%$ to $60 \%$ in the elution solvent resulted in an increase in the recovery of DON from $15.0 \%$ to $76.9 \%$, while $80 \%$ and $100 \%$ methanol in water gave recoveries of $99.7 \%$ and $100.4 \%$, respectively. Therefore, a higher methanol content $(>80 \%)$ could effectively desorb DON from the IMB, which was consistent with a reported study on IAC [26]. However, the purified DON in the eluent could not be directly injected onto the UPLC-UV system prior to evaporation and redissolution with the mobile phase, because the high quantity of methanol $(>30 \%)$ rendered the peak broad, as shown in Figure 3d. However, when the methanol concentration was lower than $100 \%$, the speed of drying by nitrogen blowing was obviously slower than that for $100 \%$ methanol, due to the presence of water. Therefore, $100 \%$ methanol was more suitable as the eluent.

\subsubsection{Elution Ability of Hot Water}

Currently, the eluents used for IAC in application notes from manufacturers are organic solvents (either methanol or acetonitrile), which are usually not injected directly into the reversed-phase chromatographic system. The eluent was further diluted or evaporated and redissolved in the mobile phase to avoid peak broadening $[5,13]$. Hence, the idea to investigate an organic solvent-free eluent was initially triggered by using hot water to disrupt the affinity of the antibodies [3,27]. The cooled hot water eluent could be directly injected onto a reversed-phase liquid chromatography column without evaporation, 
reconstitution or dilution. Hence, the aim of this study was to automatically implement hot water as the eluent of IMB for DON, replacing organic solvents in the standard elution protocol. Elution temperature is a critical parameter to ensure quantitative elution of DON from IMB. When the IMB was transferred to the eluting well, it was automatically heated to the set temperature and kept there for $5 \mathrm{~min}$. Finally, the eluent was further analyzed by UPLC. Recoveries of DON at different temperatures (Figure 3c) revealed that elution at $70{ }^{\circ} \mathrm{C}$ was ineffective (less than $20 \%$ ), elution at $80^{\circ} \mathrm{C}$ was still incomplete (in the range of $80 \%$ to $90 \%$ ), and DON was quantitatively eluted at $90{ }^{\circ} \mathrm{C}$. Hence, DON could be extracted and cleaned up by pure water, with no need for any organic solvent prior to chromatographic determination.

\subsection{The Selectivity and Specificity of the IMB}

The selectivity and specificity of the IMB toward DON was assessed by comparing the recoveries of $\mathrm{DON}$, popular mycotoxins (i.e., aflatoxin $\mathrm{B}_{1}\left(\mathrm{AFB}_{1}\right)$, aflatoxin $\mathrm{M}_{1}\left(\mathrm{AFM}_{1}\right)$, aflatoxin $B_{2}\left(A F B_{2}\right)$, aflatoxin $G_{1}\left(A F G_{1}\right)$, aflatoxin $G_{2}\left(A_{F G}\right)$, deoxynivalenol-3-glucoside (DON-3G), fumonisin $\mathrm{B}_{1}\left(\mathrm{FB}_{1}\right)$, fumonisin $\mathrm{B}_{2}\left(\mathrm{FB}_{2}\right)$, fumonisin $\mathrm{B}_{3}\left(\mathrm{FB}_{3}\right)$, nivalenol (NIV), ochratoxin A (OTA), sterigmatocystin (ST), T-2 toxin (T-2), zearalenone (ZEN)) and the structural analogs of DON (3-acetyl-deoxynivalenol (3-ADON), 15-acetyl-deoxynivalenol (15-ADON) and deoxynivalenol-3-glucoside (DON-3G)). Following the procedure outlined in "4.5. Preparation of anti-DON immunoaffinity magnetic beads," the anti-DON mAb was substituted with BSA (0.2\% BSA, 0.1 M MES, $0.15 \mathrm{M} \mathrm{NaCl}, \mathrm{pH}$ 6.0) and tris $(50 \mathrm{mM}$ Tris- $\mathrm{HCl}, 0.15 \mathrm{M} \mathrm{NaCl}, \mathrm{pH}$ 7.2) to produce BSA-MB and Tris-MB. The nonspecific test of $\mathrm{MB}$ was, respectively, conducted with BSA-MB and Tris-MB for the recoveries of the above 17 kinds of mycotoxins. As illustrated in Figure 4a by UHPLC-HRMS, the recoveries of ST and ZEN were in the range of $10-20 \%$ for both BSA-MB and Tris-MB and $70-80 \%$ of OTA for BSA-MB, while they were lower than $10 \%$ for the other mycotoxins. Through comparison, the 70\% recovery of OTA was due to the affinity of BSA to OTA [28], while the Tris-MB had little nonspecific adsorption for common mycotoxins. Hence, $50 \mathrm{mM}$ Tris- $\mathrm{HCl}, 0.15 \mathrm{M} \mathrm{NaCl}, \mathrm{pH} 7.2$ was utilized to block the remaining active sites of MB. Secondly, to further demonstrate the selective detection of DON, the above 17 kinds of mycotoxins, except DON, were chosen as the negative controls for the anti-DON IMB. The eluent in the 7-well was evaluated by UHPLC-HRMS. As shown in Figure 4a, the recoveries of DON and 15-ADON were approximately $100 \%$, while those of the other toxins were lower than 10\%, which were in accordance with a previous report [1]. When the DON immunogen was synthesized by coupling the carrier protein (BSA) via a linker on C3 and/or C15 of DON before immunization, the generated monoclonal antibody could recognize DON and 15-ADON, but not 3-ADON. Fortunately, this does not interfere with the quantification of DON, owing to their different retention times (3.350 min for DON and $17.997 \mathrm{~min}$ for 15- ADON in Figure 4b). Finally, the comparison of the chromatograms of the DON standard, blank wheat and corn extracts and the eluting solution of the positive wheat and corn extracts in Figure 4 a shows no interference peak at the retention time of DON, which was clearly separated with good peak shapes. Consequently, anti-DON IMB could effectively remove matrix interference in real samples, with acceptable selectivity and specificity. 


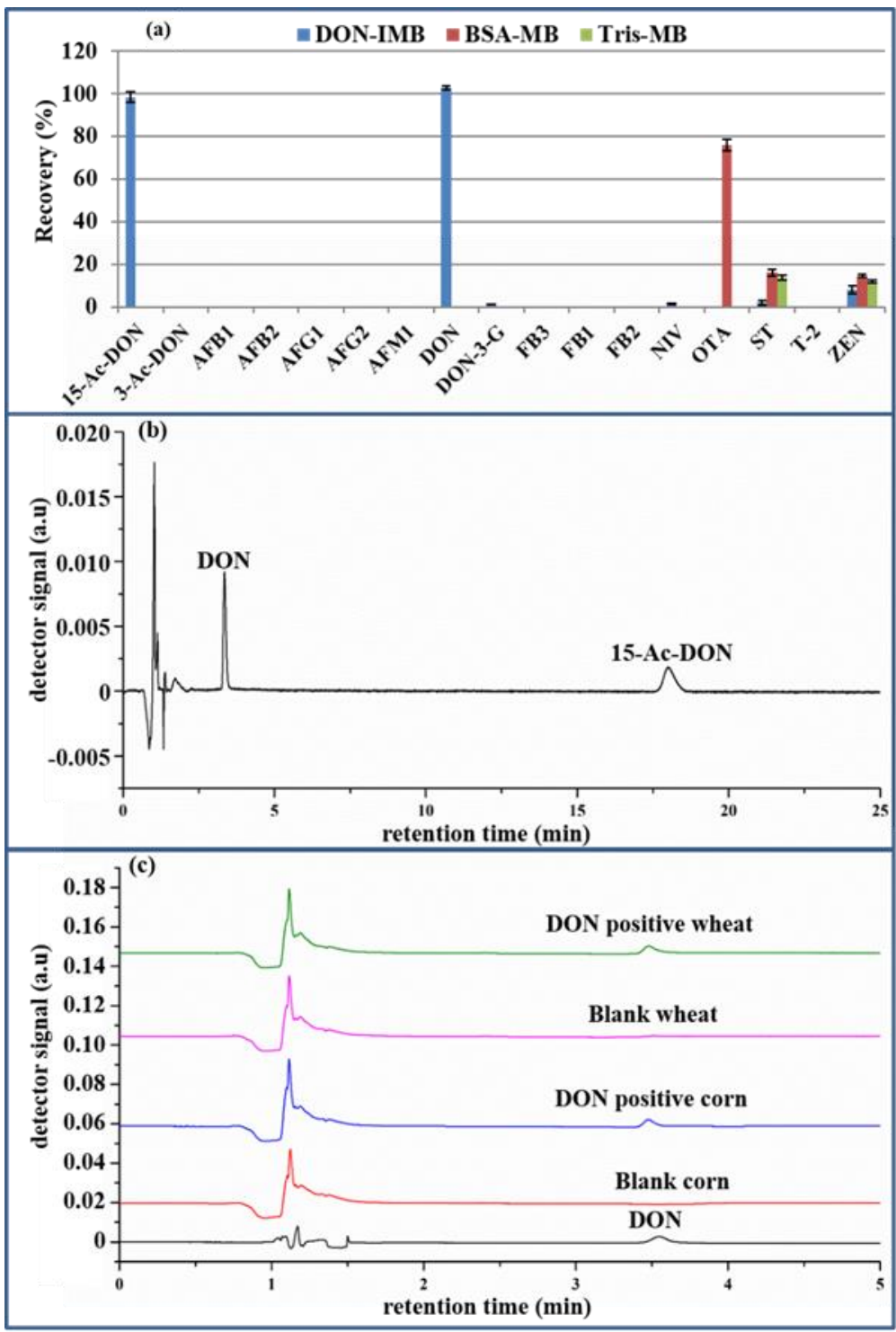

Figure 4. The recovery of the 17 kinds of mycotoxins pretreated with BSA-MB, Tris-MB and anti-DON IMB (a), the chromatogram of DON and 15-ADON (b) and the chromatogram of the DON standard, blank wheat and corn extracts and the positive wheat and corn extracts (c). (DON: deoxynivalenol; 15-ADON: 15-acetyl-deoxynivalenol BSA-MB: bovine serum albumin-magnetic bead; Tris-MB: Trismagnetic bead; IMB: immunoaffinity magnetic bead).

\subsection{Analytical Performance}

The performance of the developed method was thoroughly verified according to EU Regulation (No. 401/2006) [29], including linearity, LOD, LOQ, accuracy and precision. The peak area of various concentrations of DON detected by UPLC-UV obviously rose as its concentrations increased. A six-point calibration curve $(y=60.091 x+650.92$, $\left.\mathrm{R}^{2}=0.9999\right)$ was generated between the peak area $(y)$ and DON concentration $(x)$ in the range of $25-1500 \mathrm{ng} / \mathrm{mL}$. The LODs (three times of the background chromatographic noise) and LOQs (10 times of the background chromatographic noise) [23] were $40 \mu \mathrm{g} / \mathrm{kg}$ and $100 \mu \mathrm{g} / \mathrm{kg}$, respectively, which demonstrated the excellent sensitivity of this method. The recovery rate was used to characterize the accuracy of the analytical results, while the relative standard deviation (RSD) was used to characterize the method's precision. The detected amount and recoveries $(n=3)$ in corn and wheat were shown in Table 1 at four distinct spiking levels. Recoveries ranged from $92.0 \%$ to $109.5 \%$, while the RSD was $2.1 \%$ to $7.0 \%$, indicating good accuracy and precision. Hence, the aforesaid method for 
validation of data met all of the requirements of EU Regulation (No. 401/2006) [29], which means that the current IMB-based automatic pretreatment coupled with the UPLC-UV method was selective, precise and accurate.

Table 1. Detected amount and recovery of corn and wheat at different spiking levels $(n=3)$ (RSD: relative standard deviations).

\begin{tabular}{|c|c|c|c|c|}
\hline Matrix & $\begin{array}{l}\text { Spiked Level } \\
(\mu \mathrm{g} / \mathrm{kg})\end{array}$ & $\begin{array}{c}\text { Detected Amount } \\
(\mu \mathrm{g} / \mathrm{kg})\end{array}$ & Recovery (\%) & RSD (\%) \\
\hline \multirow{4}{*}{ corn } & 100 & $98.4 \pm 6.9$ & $98.4 \pm 6.9$ & 7.0 \\
\hline & 500 & $485.8 \pm 28.0$ & $97.2 \pm 5.6$ & 5.8 \\
\hline & 1000 & $919.6 \pm 51.3$ & $92.0 \pm 5.1$ & 5.6 \\
\hline & 2000 & $1840.1 \pm 80.8$ & $92.0 \pm 4.0$ & 4.4 \\
\hline \multirow{4}{*}{ wheat } & 100 & $109.5 \pm 4.2$ & $109.5 \pm 4.2$ & 3.8 \\
\hline & 500 & $473.1 \pm 22.4$ & $94.6 \pm 4.5$ & 4.7 \\
\hline & 1000 & $927.2 \pm 19.1$ & $92.7 \pm 1.9$ & 2.1 \\
\hline & 2000 & $1922.3 \pm 120.7$ & $96.1 \pm 6.0$ & 6.3 \\
\hline
\end{tabular}

\subsection{Application in Real Samples}

With the aim of validating the feasibility and applicability, 30 batches of real samples collected from local stores and markets were determined by the suggested approach under optimum settings. The extracted solution of these real samples was pretreated with IMB and IAC, and the eluent was quantified by UPLC-UV. A positive correlation $\left(y=0.8212 x+162.09, \mathrm{R}^{2}=0.9744\right)$ of the detected amount above LOQ from IMB and IAC was obtained in Figure 5, which means that the results from IMB agree well with those from the IAC method. Furthermore, the feasibility and applicability of the IMB was verified with CHARM DON reference materials, including MRM-DON-CORN-0.5-0021, MRM-DON-CORN-1-002G, MRM-DON-WHEAT-0.5-005A, and MRM-DON-WHEAT-1010. The detected DON by UPLC-UV is shown in Table 2, which also agrees well with the assigned value, in accordance with the National Measurement Standards of China (JJF 1507-2015) [30] and the ISO Guide 33:2015 [31]. The above results indicated that the proposed method was very suitable for the determination of DON in real samples. These results further confirm that IMB could be used as a new preprocessing platform for rapid purification and concentration assays of DON.

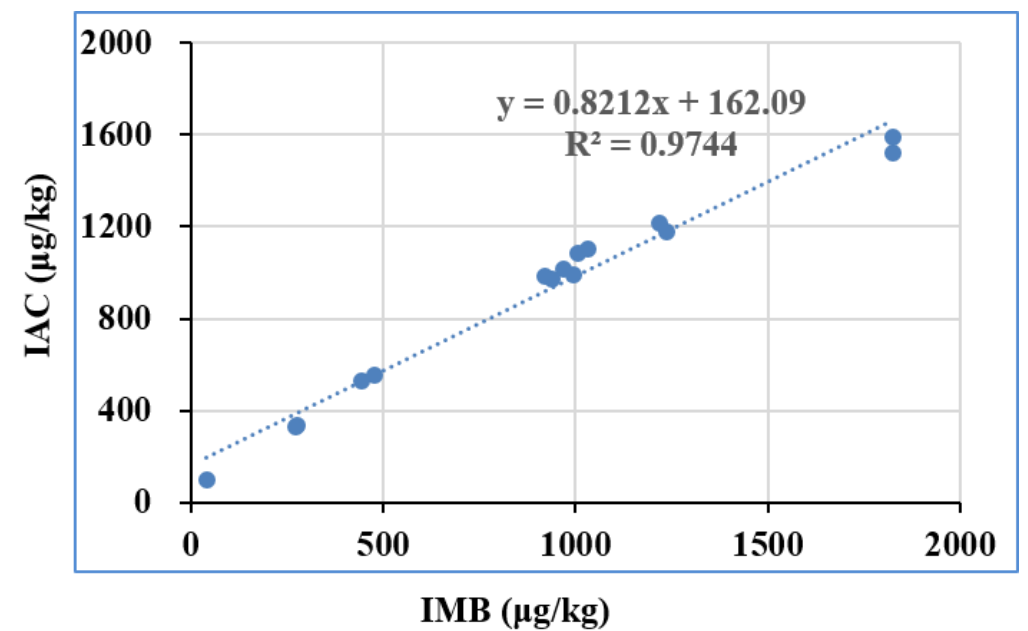

Figure 5. Comparison of the IMB method with the standard IAC method based on tests on real samples (IMB: immunoaffinity magnetic bead). 
Table 2. Detected amount of the deoxynivalenol reference materials $(n=3)$.

\begin{tabular}{cccc}
\hline $\begin{array}{c}\text { DON Reference } \\
\text { Material }\end{array}$ & Lot Number & $\begin{array}{c}\text { Detected Amount } \\
(\boldsymbol{\mu g} / \mathbf{k g})\end{array}$ & $\begin{array}{c}\text { State Value ( } \pm, \text { SD, } \\
\boldsymbol{\mu g} / \mathbf{k g})\end{array}$ \\
\hline \multirow{2}{*}{ corn } & MRM-DON-CORN-0.5-0021 & 510.4 & $520 \pm 100$ \\
& MRM-DON-CORN-1-002G & 986.7 & $1090 \pm 20$ \\
\multirow{2}{*}{ wheat } & MRM-DON-WHEAT-0.5-005A & 492.4 & $510 \pm 20$ \\
& MRM-DON-WHEAT-1-010 & 943.9 & $1000 \pm 40$ \\
\hline
\end{tabular}

\section{Conclusions}

An automated pretreatment method based on anti-DON IMB coupled with UPLC-UV analysis was successfully developed and implemented for the quantification of DON in naturally contaminated samples obtained from local markets in China as well as in certified reference material. NHS-MBs with excellent magnetic responsivity and dispersibility were synthesized and characterized, serving as carriers of anti-DON mAb. The antibodies were covalently bound to prepare IMB without the need for any activation reagent. Water, rather than the usual methanol or acetonitrile, was utilized to extract DON from real samples, as well as to elute DON from IMB, after meticulously tuning the binding and elution conditions. Hence, the proposed method is not only green and environmentally safe, but also eliminates the need for dilution or evaporation prior to UPLC-UV analysis. DON in 20 corn and wheat samples were analyzed in the range of $100 \mu \mathrm{g} / \mathrm{kg}$ to $2680 \mu \mathrm{g} / \mathrm{kg}$ by MAPI. The overall procedure for automatic handling of 20 samples took 30 mins demonstrating the simple and high throughput character of the proposed clean-up procedure. Under the optimal conditions, the developed method has good linearity, LODs, LOQs, accuracy, precision, and selectivity. Comparing results obtained with classic IAC clean-up and the developed IMB when analyzing commercial samples demonstrated the reliability and usability of IMB in high-throughput applications. Furthermore, this automatic pretreatment method provides a reference for other mycotoxins, environmental pollutants and pesticide residues.

\section{Materials and Methods}

\subsection{Chemicals and Regents}

Sigma-Aldrich (St. Louis, MI, USA) provided N-Hydroxysuccinimide and N-(3dimethylamnopropyl)-N-ethylcarbodiimide hydrochloride (EDC). Morpholinoethanesulfonic acid (MES) monohydrate was provided by shanghai macklin Biochemical Technology Co., Ltd. (Shanghai, China), while bovine serum albumin (BSA) was by beijing biotopped Technology Co., Ltd. (Beijing, China). The HPLC grade of methanol (MeOH) and acetonitrile (ACN) were purchased from Fisher Scientific (Waltham, MA, USA). The Milli-Q purification system (Bedford, MA, USA) provided ultrapure water. The purified anti-DON monoclonal antibody $(\mathrm{mAb})$ at $3.15 \mathrm{mg} / \mathrm{mL}$ was prepared by Wuxi Chuangpu Biological Technology Co., Ltd. (Jiangsu, China) in phosphate-buffered saline (PBS). The stock standard solution of DON and other mycotoxin standards in amber glass vials at $-20{ }^{\circ} \mathrm{C}$ were bought from Romer Labs, Inc. (Union, MO, US), including 3-acetyl-deoxynivalenol (3-ADON), 15-acetyl-deoxynivalenol (15- $\mathrm{ADON})$, aflatoxin $\mathrm{B}_{1}\left(\mathrm{AFB}_{1}\right)$, aflatoxin $\mathrm{M}_{1}\left(\mathrm{AFM}_{1}\right)$, aflatoxin $\mathrm{B}_{2}\left(\mathrm{AFB}_{2}\right)$, aflatoxin $\mathrm{G}_{1}\left(\mathrm{AFG}_{1}\right)$, aflatoxin $\mathrm{G}_{2}\left(\mathrm{AFG}_{2}\right)$, deoxynivalenol-3-glucoside (DON-3G), fumonisin $\mathrm{B}_{1}\left(\mathrm{FB}_{1}\right)$, fumonisin $\mathrm{B}_{2}\left(\mathrm{FB}_{2}\right)$, fumonisin $\mathrm{B}_{3}\left(\mathrm{FB}_{3}\right)$, nivalenol (NIV), ochratoxin A (OTA), sterigmatocystin (ST), T-2 toxin (T-2), zearalenone (ZEN). The above stock solution was diluted with $50 \%$ methanol/water solution to prepare the corresponding working standard solutions, which were kept in amber glass vials at $2-8{ }^{\circ} \mathrm{C}$ for a month and then renewed. The DON reference material (lot number: MRM-DON-CORN-0.5-0021, MRM-DON-CORN-1-002G, MRM-DON-WHEAT-0.5-005A, MRM-DON-WHEAT-1-010) was provided by Charm Sciences, Inc. (MA, USA). The $0.2-\mu \mathrm{m}$ PTFE acrodisc Syringe Filters were provided by PALL (Port Washington, NY, USA). Ready-to-use PBS powder (10 mM, $\mathrm{pH}$ 7.4) was purchased from Boster Biological Technology Co. LTD (Wuhan, China). All additional analytical-grade chemical reagents, such as tetraethoxysilane, polyethylene glycol 8000 (PEG 8000), epichlorohydrin, iron chloride hexahydrate $\left(\mathrm{FeCl}_{3} \cdot 6 \mathrm{H}_{2} \mathrm{O}\right)$, glycine, and 
agarose were provided by Shanghai Aladdin Biochemical Technology Co., LTD (Shanghai, China). To minimize DON pollution in the environment, all DON-contaminated laboratory glassware, containers and waste were soaked in 5\% sodium hypochlorite for more than $24 \mathrm{~h}$, prior to further washing with detergent and water, or discarding.

\subsection{Instrument and Analytical Conditions}

MTV-100 multitube vortexer (Hangzhou Aisheng instrument Co., Ltd., Hangzhou, China) was used to extract DON from samples at $2500 \mathrm{rpm}$ for $20 \mathrm{~min}$. Mettler toledo International Trading Co., LTD (Shanghai, China) provided the FE28 pH meter, and Sigma Laborzentrifugen GmbH (Osterode am Harz, Germany) provided the refrigerated centrifuge. The absorbance at $260 \mathrm{~nm}$ was measured by an ultraviolet-visible spectrophotometer (SMA4000, Merinton Instrument Inc., Beijing, China). The topology, size, and optical morphology of the MB and IMB were, respectively, studied by both an SU-70 scanning electron microscope (SEM) (Tokyo, Japan) and 6XB upright metallurgical microscopy (Shanghai optical instrument manufacture, China). The laser diffraction method (Mastersizer 3000, Malvern Inc., Malvern, UK) was used to record the particle size and distribution of the IMB.

The 10.0 $\mu \mathrm{L}$ of samples and DON standards were analyzed by Waters ACQUITY UPLC H-class system, equipped with a binary pump, an inline degasser vacuum degasser and a UV detector at $218 \mathrm{~nm}$, which was controlled by Waters EMPOWER 3 software (Waters, Manchester, UK). Chromatographical separation at $40{ }^{\circ} \mathrm{C}$ was conducted with a $\mathrm{BEH}$ reverse-phase $\mathrm{C} 18$ column $(2.1 \mathrm{~mm} \times 100 \mathrm{~mm}, 1.7 \mu \mathrm{m})$ (Waters, Manchester, UK). Iisocratic elution was carried out with acetonitrile-water $(10: 90, v / v)$ as the mobile phase in $0.3 \mathrm{~mL} / \mathrm{min}$.

Ultra-high-performance liquid chromatography coupled with high-resolution mass spectrometry (UHPLC-HRMS) was utilized to investigate the specificity of anti-DON IMB, including an UHPLC system (Ultimate 3000, Thermo Fisher Scientific, Waltham, MA, USA) and an Orbitrap mass spectrometer (Exactive ${ }^{\mathrm{TM}}$; Thermo Fisher Scientific, Waltham, MA, USA). The temperature of a CORTECS UHPLC C18 column $(100 \mathrm{~mm} \times 2.1 \mathrm{~mm}$, $1.6 \mu \mathrm{m}$ ) (Waters, Milford, MA, USA) was set at $40^{\circ} \mathrm{C}$, with an injection volume of $2 \mu \mathrm{L}$. The mobile phase was set at a flow rate of $0.3 \mathrm{~mL} / \mathrm{min}$, including $0.1 \%$ formic acid and $1 \mathrm{mM}$ ammonium acetate $(\mathrm{A})$, and $\mathrm{MeOH}(\mathrm{B})$. The following was the gradient mode: $0-2 \mathrm{~min}$ : 10\% B; 3 min: 20\% B; 4 min: 21\% B; 5-7 min: 26\% B; 10.5-13.5 min: 60\% B; $14.5-17$ min: $95 \%$ B; 18-21 min: 10\% B. MS spectra equipped with an electrospray ion (ESI) source in positive ionization mode was acquired in the full mass scan mode in the mass range of $100-800 \mathrm{~m} / \mathrm{z}$, with the resolving power set at full-width at half maximum of 70,000 . The following are the detailed operational parameters: $300{ }^{\circ} \mathrm{C}$ and $320^{\circ} \mathrm{C}$ for source temperature and capillary temperature, 35 and 10 arbitrary units for sheath gas and arbitrary units, and $3.2 \mathrm{kV}$ for capillary voltage. The precise $\mathrm{m} / \mathrm{z}$ values for the extracted ions of the 17 mycotoxins in the UHPLC-HRMS are listed in Table S1, as described in our previous reports [32].

\subsection{Synthesis of Magnetic Beads}

The magnetic beads centered on $\mathrm{Fe}_{3} \mathrm{O}_{4} \cdot \mathrm{SiO}_{2}$ and wrapped in hydrophilic agarose were synthesized as described in our previous study [20], as shown in the Electronic Supporting Material (ESM).

\subsection{Synthesis of N-Hydroxysuccinimide-Terminated Magnetic Beads}

The MB was first modified with epoxy groups and then further activated by carboxyl groups with the aid of glycine, according to our previous method [20], which was also shown in the ESM. Finally, the carboxyl-activated MB was further functionalized with an NHS group according to a previously published approach, with a few tweaks [33,34].

Next, $1 \mathrm{~mL}$ of the carboxylated MBs was, firstly, washed extensively in anhydrous DMSO 3 times to achieve anhydrous conditions. The carboxylic group on the surface of MBs was then activated at $45^{\circ} \mathrm{C}$ for $3 \mathrm{~h}$ with $200 \mathrm{mg}$ EDC and $200 \mathrm{mg}$ NHS. After 
incubation, the NHS-terminated MB was washed 3 times with anhydrous DMSO solution and stored in anhydrous N-N-dimethyl acetamide (DMAC) to prevent hydrolysis of the NHS group.

\subsection{Preparation of Anti-DON Immunoaffinity Magnetic Beads}

The anti-DON mAb was coupled with NHS-terminated MB with some modifications, according to previously published methods [35]. With the use of an external magnetic field, $20 \mathrm{~mL}$ of NHS-terminated MB $(10 \%, v / v)$ was mixed uniformly and rinsed twice with absolute ethanol. Then, $40 \mathrm{mg}$ of anti-DON mAb $(3.15 \mathrm{mg} / \mathrm{mL}$, in PBS) was diluted with coupling buffer ( $0.1 \mathrm{M} \mathrm{MES}$ and $0.15 \mathrm{M} \mathrm{NaCl}$ in $\mathrm{pH} 6.0)$ to $40 \mathrm{~mL}$, and then rotated at $20 \mathrm{rpm}$ with NHS-MB for $2 \mathrm{~h}$ at room temperature. Afterward, $40 \mathrm{~mL}$ of blocking buffer (0.05 M Tris- $\mathrm{HCl}$ and $0.15 \mathrm{M} \mathrm{NaCl}$ in $\mathrm{pH} 7.2)$ was utilized to block the remaining NHS groups of the MB under the same conditions. Finally, the anti-DON IMB was washed twice with $40 \mathrm{~mL}$ washing buffer (PBS with $0.1 \%$ Tween 20 in pH 7.2), $40 \mathrm{~mL}$ PBS three times, and then resuspended in $20 \mathrm{~mL}$ PBS with $0.02 \%$ of sodium azide at $4{ }^{\circ} \mathrm{C}$ for further use.

\subsection{Sample Sources and Preparation}

Agricultural products, including wheat and corn, were bought in local stores and supermarkets, the representative samples of which were finely milled and thoroughly homogenized in accordance with the general guidelines from the FAO and WHO about sampling [36]. Samples were extracted according to national standard GB 5009.111-2016, with some modifications [37]. Next, $5 \mathrm{~g}$ of representative sample, $1 \mathrm{~g}$ of PEG 8000 and $20 \mathrm{~mL}$ of ultrapure water were thoroughly vortexed at $2500 \mathrm{rpm}$ for $20 \mathrm{~min}$ by a multitube vortexer in a centrifuge tube of $50 \mathrm{~mL}$, which was subsequently centrifuged at $8000 \mathrm{rpm}$ for $5 \mathrm{~min}$ before the clean-up operation.

\subsection{IMB Purification}

The DON purification kit was developed on the basis of anti-DON IMB with a specially tailored 7-tube strip as the reaction vessel. The MAPI with an intelligent mechanical unit was used to automatically perform the whole DON purification process. Under the coordinated control of an embedded real-time operating system, it can not only simultaneously purify and concentrate DON from 1 to 20 samples in parallel with the DON purification kit, but also meet the requirement for rapid sample processing with a lightweight design. The corresponding magnetic bar on the instrument is a quick, simple and efficient way to aggregate the IMB to the outer wall of the magnetic bar sleeve from the reaction liquid [38]. The purification and concentration of DON can be achieved in only 3 steps, by immobilizing the liquid and transferring the magnetic beads, with no need for professional technicians. Firstly, the supernatant $(1 \mathrm{~mL})$ was added into the first well of the kit. Secondly, the kit was put into the mycotoxin automatic purification instrument, the purification program for DON was selected and the "Run" button was clicked. The optimized purification program was as follows. Briefly, the IMB was mixed for $1 \mathrm{~min}$ by moving the magnetic bar sleeve on the mechanical arm up and down. After magnetic separation for $1 \mathrm{~min}$, it was mixed in the incubation well for 5 min by the moving magnetic bar sleeve left and right. Then, the IMB captured with DON was recaptured with a magnetic bar to aggregate to the outer wall of the magnetic bar sleeve from the reaction liquid. The IMB with DON was released and recaptured after suspension in the two wash wells for $1 \mathrm{~min}$. After the IMB with DON was transferred to the eluting well and eluted for $5 \mathrm{~min}$ at $100{ }^{\circ} \mathrm{C}$, the IMB was recaptured and released in the second well. Finally, the eluent was accurately removed and filtered through a $0.22 \mu \mathrm{m}$ PTFE syringe filter before being examined using the UPLC-UV system.

\subsection{Immunoaffinity Column Purification}

The main operation steps of IAC for DON were summarized as follows, following the manufacturer's instructions and a reported study [3]. Firstly, $2 \mathrm{~mL}$ of the supernatant from "4.6. Sample sources and preparation" was flowed through the IAC at a rate of 
2-3 $\mathrm{mL} / \mathrm{min}$ to ensure the adequate capture of DON by the IAC. Subsequently, the IAC was, successively, rinsed with $10 \mathrm{~mL}$ of PBS, $10 \mathrm{~mL}$ of distilled water, and then $2-3 \mathrm{~mL}$ of flushing air. Finally, $1 \mathrm{~mL}$ methanol followed by 2-3 mL flushing air were used to elute the DON captured by IAC. The collected eluent was dried at $40^{\circ} \mathrm{C}$, under a gentle stream of nitrogen gas, which was then reconstituted with $1 \mathrm{~mL}$ acetonitrile/water $(10: 90, v / v)$ and filtered through a $0.22 \mu \mathrm{m}$ PTFE syringe filter prior to addition to the UPLC-UV system.

\subsection{Method Validation}

The developed method was validated in-house in terms of linearity, limit of detection (LOD), limit of quantification (LOQ), accuracy, precision and selectivity, according to the methods of sampling and analysis in EC Regulation (No. 401/2006) [29]. Linearity was determined with six concentrations of DON between 50 and $1000 \mathrm{ng} / \mathrm{mL}$ (50, 100, 250, $500,750,1000 \mathrm{ng} / \mathrm{mL})$ and the regression equation including the regression coefficient $\left(\mathrm{R}^{2}\right)$ was calculated by plotting the peak area $(y)$ against the concentration of DON $(x)$. LOD and LOQ were estimated in triplicate by serially diluted DON standard solution, which produced a chromatogram peak, with 3 and 10 signal-to-noise ratios, respectively. The recovery investigation in triplicate looked into the method's accuracy and precision, which was conducted by spiking the blank cereals (wheat, maize and husked rice) with various amounts of DON standards $(100,500,1000,2000 \mu \mathrm{g} / \mathrm{kg})$. The result of the recovery study was expressed as recovery rate (\%), while the relative standard deviation (RSD, \%) was used for precision. Furthermore, the DON reference material from CHARM was used to verify the accuracy of the method. Matrix interference was investigated by comparing chromatograms of DON standard, blank or positive wheat and corn extracts. The selectivity was investigated by 17 common mycotoxins including $\mathrm{AFB}_{1}, \mathrm{AFB}_{2}, \mathrm{AFG}_{1}, \mathrm{AFG}_{2}$, $\mathrm{AFM}_{1}, \mathrm{FB}_{1}, \mathrm{FB}_{2}, \mathrm{FB}_{3}, \mathrm{NIV}$, OTA, ST, T-2 and ZEN, with the aid of UHPLC-HRMS. Matrix interference was investigated by contrast chromatograms of the DON standard, blank or positive wheat and corn extracts.

\subsection{Data Management}

Data were acquired and analyzed using Waters EMPOWER3 software (Waters, Manchester, UK). Statistical analyses during the whole study were performed using Microsoft Excel and the software package OriginPro version 8.5 (OriginLab Corporation, Northampton, USA). The figures were plotted using OriginPro version 8.5.

Supplementary Materials: The following are available online at https: / www.mdpi.com/article/ $10.3390 /$ toxins14020093/s1, Table S1. Retention times and accurate masses of selected ions for 18 mycotoxins.

Author Contributions: Conceptualization, J.Y. and S.W.; Methodology, J.C., H.L. and Z.X.; Validation, H.L. and Z.L.; Formal Analysis, H.L., Y.W. and L.L.; Resources, Y.W. and L.L.; Data Curation, H.L. and J.Y.; Writing-Original Draft Preparation, M.W., S.F. and H.L; Writing-Review and Editing, J.Y. and R.K.; Supervision, S.W.; Project Administration, J.Y. and S.W.; Funding Acquisition, J.C., H.L, J.Y. and M.W. All authors have read and agreed to the published version of the manuscript.

Funding: This research was funded by the Beijing Municipal Natural Science Foundation (7192026), the Fundamental Research Funds for the Academy of National Food and Strategic Reserves Administration (ZX1922, ZX2001, ZX2009), Innovation and Capacity-building Projects by Beijing Academy of Agriculture and Forestry Sciences (KJCX20200201), the National Natural Science Foundation of China (81803712, 31901806), and the Key R\&D Program of Guangxi (AB20238018).

Institutional Review Board Statement: Not applicable.

Informed Consent Statement: Not applicable.

Acknowledgments: We thank the following people for the contribution to interlaboratory study: Mengyao Zheng, Yu Wu, Yuting Zou, Shuqing Gao, Tongtong Liu and Ziyi Jiao from Academy of National Food and Strategic Reserves Administration.

Conflicts of Interest: The authors declare no conflict of interest. 


\section{References}

1. Sanders, M.; Guo, Y.R.; Iyer, A.; García, Y.R.; Galvita, A.; Heyerickd, A.; Deforce, D.; Risseeuw, M.D.P.; Calenbergh, S.V.; Bracke, M.; et al. An immunogen synthesis strategy for the development of specific anti-deoxynivalenol monoclonal antibodies. Food Addit. Contam. Part A 2014, 31, 1751-1759. [CrossRef] [PubMed]

2. Xu, L.L.; Wen, Y.Q.; Liu, Y.L.; Ma, Y.X. Occurrence of deoxynivalenol in maize germs from North China Plain and the distribution of deoxynivalenol in the processed products of maize germs. Food Chem. 2018, 266, 557-562. [CrossRef] [PubMed]

3. Gonçalves, C.; Mischke, C.; Stroka, J. Determination of deoxynivalenol and its major conjugates in cereals using an organic solvent-free extraction and IAC clean-up coupled in-line with HPLC-PCD-FLD. Food Addit. Contam. Part A 2020, 37, 1765-1776. [CrossRef] [PubMed]

4. Mishra, S.; Ansari, K.M.; Dwivedi, P.D.; Pandey, H.P.; Das, M. Occurrence of deoxynivalenol in cereals and exposure risk assessment in Indian population. Food Control 2013, 30, 549-555. [CrossRef]

5. Park, J.; Chang, H.; Kim, D.G.; Chung, S.; Lee, C. Long-Term Occurrence of Deoxynivalenol in Feed and Feed Raw Materials with a Special Focus on South Korea. Toxins 2018, 10, 127. [CrossRef] [PubMed]

6. FAO; WHO. Safety Evaluation of Certain Mycotoxins in Food. Prepared by Fifty-Sixth Meeting of the Joint FAO/WHO Expert Committee on Food Additives (JECFA); WHO food additives Series 47; International Programme on Chemical Safety, Word Health Organization: Geneva, Switzerland, 2001; p. 74.

7. Wan, D.; Huang, L.; Pan, Y.; Wu, Q.; Chen, D.; Tao, Y.; Wang, X.; Liu, Z.L.; Li, J.; Wang, L.Y.; et al. Metabolism, distribution, and excretion of deoxynivalenol with combined techniques of radiotracing, high-performance liquid chromatography ion trap time-of-flight mass spectrometry, and online radiometric detection. J. Agric. Food Chem. 2014, 62, 288-296. [CrossRef]

8. International Agency for Research on Cancer; World Health Organization. Some naturally occurring substances, food items and constituents. Heterocyclic Aromatic Amines and Mycotoxins. Int. Agency Res. Cancer Monogr. Eval. Carcinog. Risks Hum. 1993, 56, 397-444.

9. Amuzie, C.J.; Pestka, J.J. Suppression of insulin-like growth factor acid-labile subunit expression a novel mechanism for deoxynivalenol-induced growth retardation. Toxicol. Sci. 2010, 113, 412-421. [CrossRef]

10. European Commission. Commission regulation no 1881/2006 of 19 December 2006 setting maximum levels for certain contaminants in foodstuffs. Off. J. Eur. Union L. 2006, 364, 5.

11. National Health and Family Planning Commission; China Food and Drug Administration. Maximum Levels of Mycotoxins in Foods; National Criterion of China: Beijing, China, 2017; GB/T 2761-2017.

12. Canady, R.A.; Coker, R.D.; Egan, S.K.; Krska, R.; Kuiper Goodman, T.; Olsen, M.; Pestka, J.; Resnik, S.; Schlatter, J. Deoxynivalenol. In Safety Evaluation of certain Mycotoxins in food. Food Additives No. 47. Prepared for the Fifty-Sixth Meetings of the Joint FAO/WHO Expert Committee on Food Additives (JECFA); World Health Organization (WHO): Geneva, Switzerland, $2001 ;$ pp. $419-555$.

13. Ok, H.E.; Lee, S.Y.; Chun, H.S. Occurrence and simultaneous determination of nivalenol and deoxynivalenol in rice and bran by HPLC-UV detection and immunoaffifinity cleanup. Food Control 2018, 87, 53-59. [CrossRef]

14. Klinglmayr, C.; Nobauer, K.; Razzazi-Fazeli, E.; Cichna-Markl, M. Determination of deoxynivalenol in organic and conventional food and feed by sol-gel immunoaffinity chromatography and HPLC-UV detection. J. Chromatogr. B 2010, 878, 187-193. [CrossRef] [PubMed]

15. Zhao, Z.; Rao, Q.; Song, S.; Liu, N.; Han, Z.; Hou, J.; Wu, A. Simultaneous determination of major type B trichothecenes and deoxynivalenol-3-glucoside in animal feed and raw materials using improved DSPE combined with LC-MS/MS. J. Chromatogr. B 2014, 963, 75-82. [CrossRef] [PubMed]

16. Valle-Algarra, F.M.; Medina, A.; Gimeno-Adelantado, J.V.; Llorens, A.; Jimenez, M.; Mateo, R. Comparative assessment of solidphase extraction clean-up procedures, GC columns and perfluoroacylation reagents for determination of type B trichothecenes in wheat by GC-ECD. Talant 2005, 66, 194. [CrossRef] [PubMed]

17. Rodriguez-Carrasco, Y.; Molto, J.C.; Manes, J.; Berrada, H. Development of microextraction techniques in combination with GC-MS/MS for the determination of mycotoxins and metabolites in human urine. J. Sep. Sci. 2017, 40, 1572-1582. [CrossRef]

18. Sugita-Konsihi, Y.; Tanaka, T.; Tabata, S.; Nakajima, M.; Nouno, M.; Nakaie, Y.; Chonan, T.; Aoyagi, M.; Kibune, N.; Mizuno, K. Validation of an hplc analytical method coupled to a multifunctional clean-up column for the determination of deoxynivalenol. Mycopathologia 2006, 161, 239-243. [CrossRef]

19. Wierucka, M.; Biziuk, M. Application of magnetic nanoparticles for magnetic solid-phase extraction in preparing biological, environmental and food samples. Trends Anal. Chem. 2014, 59, 50-58. [CrossRef]

20. Liu, H.M.; Lu, A.X.; Fu, H.L.; Li, B.R.; Yang, M.H.; Wang, J.H.; Luan, Y.X. Affinity capture of aflatoxin B1 and B2 by aptamerfunctionalized magnetic agarose microspheres prior to their determination by HPLC. Mikrochim. Acta 2018, 185, 326. [CrossRef]

21. Váradi, C.; Lew, C.; Guttman, A. Rapid magnetic bead based sample preparation for automated and high throughput N-glycan analysis of therapeutic antibodies. Anal. Chem. 2014, 86, 5682-5687. [CrossRef]

22. Saçligil, D.; Şenel, S.; Yavuz, H.; Denizli, A. Purification of transferrin by magnetic immunoaffinity beads. J. Sep. Sci. 2015, 38, 2729-2736. [CrossRef]

23. Ye, J.; Xuan, Z.; Zhang, B.; Wu, Y.; Li, L.; Wang, S.; Xie, G.; Wang, S.X. Automated analysis of ochratoxin A in cereals and oil by immunoaffinity magnetic beads coupled to UPLC-FLD. Food Control 2019, 104, 57-62. [CrossRef] 
24. Xuan, Z.; Ye, J.; Zhang, B.; Li, L.; Wu, Y.; Wang, S. An Automated and High-Throughput Immunoaffinity Magnetic Bead-Based Sample Clean-Up Platform for the Determination of Aflatoxins in Grains and Oils Using UPLC-FLD. Toxins 2019, 11, 583. [CrossRef] [PubMed]

25. Miron, T.; Wilchek, M. A Spectrophotometric Assay for Soluble and Immobilized A/-Hydroxysuccinimide Esters. Anal. Biochem. 1982, 126, 433-435. [CrossRef]

26. Xu, Z.L.; Deng, H.; Lei, H.T.; Jiang, Y.M.; Campbell, K.; Shen, Y.D.; Yang, J.Y.; Wang, H.; Sun, Y.M. Development of a BroadSpecifificity Monoclonal Antibody-Based Immunoaffiffiffinity Chromatography Cleanup for Organophosphorus Pesticide Determination in Environmental Samples. Agric. Food Chem. 2012, 60, 5847-5852. [CrossRef] [PubMed]

27. Stroka, J.; Seidler, J. Immunoaffinity clean-up of mycotoxins with organic solvent-free elution. World Mycotoxin J. 2014, 7, 115-120. [CrossRef]

28. Leal, T.; Abrunhosa, L.; Domingues, L.; Venâncio, A.; Oliveira, C. BSA-based sample clean-up columns for ochratoxin A determination in wine: Method development and validation. Food Chem. 2019, 300, 125204. [CrossRef]

29. European Commission. Commission Regulation (EC) No 401/2006 of 23 February 2006 laying down the methods of sampling and analysis for the official control of the levels of mycotoxins in foodstuffs. Off. J. Eur. Union 2006, 70, 12-34.

30. National Verification Regulations and Calibration Specifications of measuring Instruments of China. In The Selection and Use of Reference Materials; State General administration of the People's Republic of China for Quality Supervision and Inspection and Quarantine: Beijing, China, 2015; JJF 1507-2015.

31. ISO Guide 33: Reference Materials-Good Practice in Using Reference Materials; International Origanization for Standardization: Geneva, Switzerland, 2015.

32. Wu, Y.; Ye, J.; Xuan, Z.H.; Li, L.; Wang, H.B.; Wang, S.S.; Liu, H.M.; Wang, S.X. Development and validation of a rapid and efficient method for simultaneous determination of mycotoxins in coix seed using one-step extraction and UHPLC-HRMS. Food Addit. Contam. Part A 2021, 38, 148-159. [CrossRef]

33. Besselink, G.A.J.; Beugleing, T.; Bantjes, A. Hydrolysis of Activated Groups and Coupling of Amino Compounds. Appl. Biochernistry Biotechnol. 1993, 43, 227. [CrossRef]

34. Cuatrecasas, P.; Parikh, I. Adsorbents for affinity chromatography. Use of N-hydroxysuccinimide esters of agarose. Biochemistry 1972, 11, 2291-2299. [CrossRef]

35. Qileng, A.; Zhu, H.S.; Liu, S.Q.; He, L.; Qin, W.W.; Liu, W.P.; Xu, Z.L.; Liu, Y.J. Machine learning: Assisted multivariate detection and visual image matching to build broad-specificity immunosensor. Sens. Actuators B Chem. 2021, 339, 129872. [CrossRef]

36. Food and Agriculture Organization (FAO)/World Health Organization (WHO). The Codex General Guidelines on Sampling-CAC/GL 50-2004; FAO: Rome, Italy; WHO: Geneva, Switzerland, 2004.

37. National Health and Family Planning Commission; China Food and Drug Administration. Determination of Deoxynivalenol and Its Acetylated Derivatives in Foods; National Criterion of China: Beijing, China, 2016; GB 5009.111-2016.

38. Chen, Z.; Wu, Y.Q.; Kang, M.; He, N.Y.; Wan, S.R.; Su, E.; Wang, L.J. Research on Automated Nucleic Acid Extraction Instrument Based on Magnetic Nanoparticles Separation. Nanosci. Nanotechnol. Lett. 2018, 10, 60-68. [CrossRef] 